\title{
Medication management on sick days
}

\section{Tom N Lea-Henry \\ Advanced trainee in nephrology}

Jonathan Baird-Gunning

Advanced trainee in general medicine

\section{Elizabeth Petzel}

Medicines information pharmacist

\section{Darren M Roberts}

Nephrologist and clinical pharmacologist

Canberra Hospital and Health Services

\section{Keywords}

acute disease, adverse drug reaction, chronic kidney disease, contraception, diabetes, drug interactions

Aust Prescr 2016;39:168-73

https://doi.org/10.18773/ austprescr.2017.057

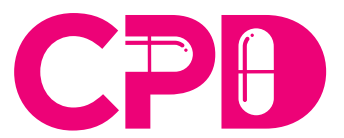

FOR PHARMACISTS

This article has a continuing professional development activity for pharmacists available at https://learn.nps.org.au

\section{SUMMARY}

Patients may be susceptible to adverse drug events during acute illness due to comorbidities or medicine use. Acute illness should prompt careful monitoring or dose adjustment in patients prescribed certain medicines.

Patient factors, severity and expected duration of illness, and class of drug should be considered to minimise the risk of adverse drug events.

Some drugs may need to be temporarily suspended, such as metformin, diuretics and sodiumglucose co-transporter 2 inhibitors when there is a risk of hypovolaemia.

Those with chronic kidney disease are at risk of acute kidney injury due to limits in their physiological reserve. This may be compounded by medicine use.

Temporary increases in dose may be required for insulin and corticosteroids.

Withdrawal syndromes may occur with controlled-release drugs, such as dopamine agonists, antidepressants and analgesics, due to malabsorption.

An action plan may be needed for medicine use on sick days. Patient education around supplemental dosing of oral contraceptives to maintain efficacy after acute illness is important.

\section{Introduction}

During intercurrent illness, the risk of an adverse drug event could be increased by ongoing use of some medicines. They may cause harm with either continued use or abrupt cessation during illness. For example, ongoing use of an antihypertensive on sick days may compound hypotension associated with the acute illness. Factors to consider for dose adjustment include the type of medicine, formulation and pharmacokinetics, duration of illness and comorbidities.

\section{Pathophysiology}

An understanding of pharmacokinetic factors associated with significant illness (sick days) can be useful to predict and manage patients at most risk. Changes in drug absorption, distribution, metabolism and excretion are well documented in critically ill patients. Unfortunately, there are less data to guide the prescribing of regular medicines in less severe illness. Common symptoms that could indicate a patient is at risk of hypovolaemia include anorexia, diarrhoea, vomiting and fever. Depending on the severity of illness and the susceptibility of the patient, volume depletion and renal dysfunction can occur. This can impair the excretion of medicines and result in accumulation and toxicity. Severe gastroenteritis may decrease the bioavailability due to reduced gut transit time and reduced drug absorption so some medications may need a corresponding increase in dose.
When the illness is short-lived and relatively minor, changes in physiology and pharmacokinetics are unlikely to be a problem for most medicines and in most patients. However, there are exceptions when patient advice about dose adjustment and monitoring for adverse outcomes may be necessary.

\section{Important comorbidities}

Patients with chronic kidney disease may be at particular risk of problems during intercurrent illness. Conditions that induce hypovolaemia increase the risk of acute kidney injury in those with reduced renal homeostatic reserve. This is potentially compounded by drugs that compromise renal homeostasis, such as renin-angiotensin system inhibitors (ACE inhibitors and sartans).

A UK position statement ${ }^{1}$ guides the management of sick days in patients at risk of acute kidney injury, particularly when there is disturbed fluid balance. It advises patients not to use renin-angiotensin system inhibitors, diuretics, non-steroidal anti-inflammatory drugs, metformin or renally excreted sulfonylureas (e.g. glibenclamide and glimepiride) when they have vomiting or diarrhoea (unless mild), or fevers, sweats and rigors.

Other comorbidities that may impede a normal homeostatic response to disturbed fluid balance are diabetes and congestive cardiac failure. In addition, the normal homeostatic hormone responses to stress can affect glycaemic control in diabetes. In 
patients with corticosteroid-induced suppression of the hypothalamic-pituitary-adrenal axis or adrenal insufficiency, the hormone response to stress may be impaired.

\section{Controlled-release formulations}

Drugs with a controlled-release formulation may be more susceptible to decreased bioavailability in severe diarrhoea. ${ }^{2}$ For some medicines, this can be associated with a withdrawal or discontinuation syndrome (see Table), ${ }^{3,4}$ which is predictable and potentially avoidable. This is a particular concern with shorter acting drugs in a controlledrelease formulation (which depends on normal gut transit time) when the decrease in plasma concentration reduces the patient's functional status (e.g. controlled-release formulations of opioids or dopamine agonists).

\section{Renin-angiotensin system inhibitors}

When blood volume is reduced, an increase in angiotensin II promotes proximal tubule sodium reabsorption, aldosterone synthesis and thirst which act together to defend renal perfusion and the glomerular filtration rate. Illnesses that cause hypovolaemia can reduce renal perfusion. Since the normal physiological response to hypovolaemia is impaired by ACE inhibitors and sartans, patients taking these drugs are at increased risk of acute kidney injury.

When preparing its guidelines, the UK National Institute for Health and Care Excellence found no relevant publications on withholding reninangiotensin system inhibitors during intercurrent illness. However, it concluded that the risk of acute kidney injury with their continued use outweighs the potential risk of cardiovascular events if they are temporarily stopped. ${ }^{5}$ The guidelines suggested that patients should be advised to suspend reninangiotensin system inhibitors during episodes of diarrhoea, vomiting and hypotension, or major infection, until they are 'clearly improving.'

The Kidney Disease Improving Global Outcomes guideline recommends monitoring renal function and serum potassium in patients taking a reninangiotensin system inhibitor during an illness that risks dehydration. ${ }^{6}$ Given the current uncertainty, it seems reasonable to withhold these drugs in the unwell patient.

While this recommendation may sound rational, the data supporting withholding of renin-angiotensin system inhibitors in other circumstances are conflicting. In a meta-analysis of observational studies in patients undergoing surgery, when there are likely to be associated changes in haemodynamics, renin-angiotensin system inhibitors increased the odds of postoperative acute kidney injury and mortality. ${ }^{7}$ However, a randomised controlled trial (in patients with normal baseline creatinine $)^{8}$ and a meta-analysis suggested reninangiotensin system inhibitors were protective. ${ }^{9}$ The more widely used renin-angiotensin system inhibitors have a reasonably prolonged half-life so a rapid offset of effect will not occur when the medicine is withheld for a few days.

The benefits from renin-angiotensin system inhibitors reflect cardiac and vascular remodelling that follows treatment over many years. Also, severe rebound hypertension does not occur following cessation of renin-angiotensin system inhibitors. Until more data are available, it is reasonable to withhold renin-angiotensin system inhibitors in patients with intercurrent illnesses associated with volume depletion until symptoms resolve.

\section{Diuretics}

Diuretics promote volume loss, which can induce renal dysfunction and change electrolytes. This may be exacerbated in patients with intercurrent illnesses. A retrospective study of older patients prescribed spironolactone in combination with an ACE inhibitor for cardiac failure found that intercurrent illness increased the risk of severe renal insufficiency and hyperkalaemia. The study recommended that spironolactone may need to be temporarily stopped during illness involving dehydration. ${ }^{10}$ In patients at risk of dehydration or hypotension, spironolactone and probably other diuretics should be temporarily withheld.

\section{Centrally acting antihypertensives}

Most antihypertensives are not associated with marked rebound hypertension or other complications (with several important exceptions) due to a transient sub-therapeutic concentration from reduced absorption, or if discontinued.

Clonidine is an agonist at alpha ${ }_{2}$ and imidazoline receptors while moxonidine predominantly acts on the I, imidazoline receptor." Both reduce blood pressure by reducing sympathetic tone. Abrupt discontinuation of clonidine is associated with rebound hypertension higher than pre-treatment pressures. ${ }^{12}$ This risk may be less with moxonidine. ${ }^{13,14}$

Rebound hypertension may be more marked if the patient is concurrently taking a beta blocker due to unopposed stimulation of alpha receptors. ${ }^{15}$ We do not advise routinely withholding clonidine or moxonidine on sick days, but blood pressure should be monitored when drug bioavailability may be reduced. 


\section{Table Drugs associated with adverse events in intercurrent illness}

\begin{tabular}{|c|c|c|c|}
\hline Drug class & Drug examples & Problems arising from intercurrent illness & Potential adverse outcome \\
\hline \multirow[t]{3}{*}{ Analgesics } & \multirow{2}{*}{$\begin{array}{l}\text { Hydromorphone, morphine, } \\
\text { oxycodone, tramadol }\end{array}$} & \multirow{2}{*}{$\begin{array}{l}\text { Reduced absorption of controlled-release } \\
\text { formulations, or deliberate cessation }\end{array}$} & Exacerbation of pain \\
\hline & & & $\begin{array}{l}\text { Opioid withdrawal syndrome - dysphoria, } \\
\text { restlessness, salivation, nausea, abdominal } \\
\text { pain and diarrhoea }\end{array}$ \\
\hline & Morphine, hydromorphone & $\begin{array}{l}\text { Reduced clearance in renal dysfunction, } \\
\text { with risk of accumulation and toxicity }\end{array}$ & Opioid toxicity \\
\hline
\end{tabular}

Antidepressants Venlafaxine, desvenlafaxine Reduced absorption of controlled-release formulations, or deliberate cessation

Withdrawal syndrome - agitation, anxiety, diarrhoea, fasciculations, sensory disturbance (including shock-like syndrome, tremor, vertigo and vomiting)

Exacerbation of angina

Conflicting data on association with rebound hypertension, arrhythmias ${ }^{3}$

Renin-angiotensin inhibitors Impaired physiological homeostasis, Acute kidney injury and hyperkalaemia
impairing renal perfusion

Diuretics Exacerbation of hypovolaemia and altered electrolyte excretion

Clonidine or moxonidine Reduced absorption or deliberate cessation leading to withdrawal of central inhibitory effect
Drugs for parkinsonism
Levodopa with carbidopa or benserazide
Reduced absorption of controlled-release formulations, or deliberate cessation
Dehydration and electrolyte disequilibria

Tachycardia and hypertension

Decline in motor function

Case reports of neuroleptic malignant syndrome with acute withdrawal ${ }^{4}$ featuring fever, altered mental state, rhabdomyolysis, rigidity

Mood stabiliser Lithium Reduced clearance in renal dysfunction with risk of accumulation and toxicity

Lithium toxicity - nausea, confusion, muscle weakness, apathy, hyperreflexia, myoclonic jerks, dysarthria, seizures

Reduced absorption or deliberate cessation

Reduced anticoagulant effect and elevated risk of thrombosis apixaban, dabigatran

Risk of accumulation in renal dysfunction

Dabigatran, rivaroxaban, apixiban

Warfarin

Decreased oral intake contributing to vitamin $\mathrm{K}$ deficiency

Warfarin, rivaroxaban, apixaban

\section{Concomitant administration of anti-} infectives that reduce drug clearance e.g. erythromycin (warfarin, rivaroxaban, apixaban), ciprofloxacin (warfarin) or fluconazole (warfarin)

Antiarrhythmics Disopyramide, flecainide, sotalol, digoxin

Reduced absorption or deliberate cessation

Sotalol, digoxin

Reduced clearance in renal dysfunction

Reduced absorption or deliberate cessation

Carbamazepine, valproate, phenytoin, levetiracetam, topiramate
Increased anticoagulant activity leading to bleeding complications

Increased anticoagulant activity leading to bleeding complications

Increased anticoagulant activity leading to bleeding complications
Antiepileptics
Reduced antiarrhythmic activity and potentially life-threatening arrhythmias

Bradycardia (sotalol and digoxin) and hyperkalaemia (digoxin)

Reduction in serum concentration and increased seizure risk 


\section{Table Drugs associated with adverse events in intercurrent illness (continued)}

\begin{tabular}{|c|c|c|c|}
\hline Drug class & Drug examples & Problems arising from intercurrent illness & Potential adverse outcome \\
\hline \multirow[t]{3}{*}{ Drugs for diabetes } & $\begin{array}{l}\text { Sodium-glucose } \\
\text { co-transporter } 2 \text { inhibitors }\end{array}$ & $\begin{array}{l}\text { Exacerbation of hypovolaemia and } \\
\text { electrolyte loss }\end{array}$ & Dehydration and electrolyte disequilibria \\
\hline & Metformin & $\begin{array}{l}\text { Reduced clearance in renal dysfunction } \\
\text { causing accumulation and toxicity }\end{array}$ & Nausea, anorexia, lactic acidosis \\
\hline & Insulin, sulfonylureas & $\begin{array}{l}\text { Inappropriate dose relative to intake and } \\
\text { hormonal counterregulatory response (insulin } \\
\text { and sulfonylureas) or reduced clearance in } \\
\text { renal dysfunction causing accumulation and } \\
\text { toxicity (glibenclamide, glimepiride) }\end{array}$ & Hypoglycaemia or hyperglycaemia \\
\hline Oral contraceptives & $\begin{array}{l}\text { Oestrogen and progestogen } \\
\text { combinations }\end{array}$ & Reduced absorption or deliberate cessation & Contraceptive failure \\
\hline
\end{tabular}

\section{Beta blockers}

Sudden discontinuation of beta blockers can cause rebound hypertension. Acute coronary syndrome has also been reported, even in patients without coronary artery disease. ${ }^{16,17}$ The risk of these events appears to be inversely related to the drug's half-life and the extent of receptor downregulation. ${ }^{18}$

\section{Drugs for diabetes}

Patients able to self-manage their diabetes medicines should be provided with a management plan for use during sick days. Patients who are not monitoring their own glucose should be advised to see their doctor when becoming ill.

\section{Insulin}

During intercurrent illness, there is generally an increased insulin requirement due to upregulation of counter-regulatory hormones, particularly cortisol, so temporary changes to the insulin dose may be required. Despite a reduced nutritional intake, insulin should not be routinely withheld in type 1 or 2 diabetes.

For type 1 diabetes, guidelines suggest that patients administer supplemental doses of short-acting insulin every 2-4 hours if blood glucose remains elevated. If there is no improvement in either blood glucose or blood ketones after two extra supplemental doses of insulin, medical review should be sought. ${ }^{19}$ Patients should increase the frequency of blood glucose monitoring and add regular blood ketone measurements if their glucometer allows.

In type 2 diabetes, patients should increase blood glucose monitoring to 3-4 times daily during acute illness. If readings are persistently above $15 \mathrm{mmol} / \mathrm{L}$ then the morning dose of long- or intermediate-acting insulin may need to be increased by $10-20 \% .{ }^{20}$ For those taking short-acting insulin, the dose will need to be adjusted based on the results of their blood glucose readings and dietary carbohydrate intake.

\section{Metformin}

Patients should withhold metformin during significant illness to reduce the risk of lactic acidosis. Although more strongly associated with its predecessor phenformin, cases of lactic acidosis have also been reported with metformin. ${ }^{21}$ Observational studies suggest that it may be more common during intercurrent illness, particularly when there is vomiting, diarrhoea and acute kidney injury. ${ }^{22}$ Metformin is unlikely to induce hypoglycaemia but it can aggravate symptoms of nausea, vomiting and diarrhoea, which may increase the risk of renal dysfunction. Vomiting and diarrhoea can be early signs of lactic acidosis and may prompt further investigation. ${ }^{22}$

\section{Sulfonylureas}

Patients with severe intercurrent illness generally have an increase in blood glucose. The use of sulfonylureas may limit hyperglycaemia and the risk of a hyperosmolar syndrome. However, patients can experience reduced blood glucose in some instances, such as when severe anorexia and gastroenteritis compromise caloric intake. Patients using sulfonylureas should be advised about the increased risk of hypoglycaemia and should have a low threshold for ceasing their sulfonylurea and seeking medical assessment should they develop low blood glucose readings. Those taking sulfonylureas or repaglinide should continue their treatment and increase blood glucose monitoring to at least twice daily (before meals, including before breakfast). This needs to be done with additional caution for glibenclamide and glimepiride, which have renally excreted active metabolites, ${ }^{23,24}$ and may require closer monitoring of blood glucose. 


\section{Glucagon-like peptide-1 analogues and dipeptidyl peptidase-4 inhibitors}

While glucagon-like peptide-1 analogues have gastrointestinal adverse effects, they are considered safe in acute illness as they are not associated with hypoglycaemia ${ }^{25}$ or complications such as lactic acidosis or renal failure. ${ }^{26}$ This is because they stimulate insulin release via a glucosedependent mechanism.

The dipeptidyl peptidase-4 inhibitors (DPP-4 or gliptins) are also considered safe. They potentiate the effect of endogenous glucagon-like peptide-1 by inhibiting its metabolism.

In patients taking either of these drug classes, acute abdominal pain as part of an intercurrent illness should prompt assessment for possible druginduced pancreatitis. ${ }^{19}$

\section{Sodium-glucose co-transporter 2 inhibitors}

Sodium-glucose co-transporter 2 (SGLT2) inhibitors have an osmotic diuretic effect and should be withheld due to the risk of worsening dehydration. They have also been associated with euglycaemic ketoacidosis and therefore may contribute to the development of diabetic ketoacidosis.

\section{Corticosteroids}

The hypothalamic-pituitary-adrenal axis is suppressed in patients taking corticosteroids ( $\geq$ prednisolone $5 \mathrm{mg} /$ day or equivalent), and the normal response to severe illness is blunted. Corticosteroid doses need to be increased to mimic the normal physiological response to prevent haemodynamic instability from a relative hypoadrenal state. For example, methods for steroid escalation in acute illness include:

- increase the dose to an equivalent of hydrocortisone $50-75 \mathrm{mg} /$ day (or prednisolone $12.5-20 \mathrm{mg} /$ day $)^{27}$

- double the dose for two days before returning to the patients' usual dose when they feel better. ${ }^{28}$

If oral therapy is compromised by severe diarrhoea or vomiting, parenteral hydrocortisone may be necessary. ${ }^{28}$ Mineralocorticoids do not need to be adjusted.

\section{Digoxin}

Vomiting and diarrhoea can contribute to digoxin toxicity by two mechanisms. First, gastroenteritis can result in hypokalaemia, which potentiates the effect of digoxin. Second, the reduced glomerular filtration rate associated with hypovolaemia reduces digoxin clearance. Digoxin toxicity can manifest as nausea and vomiting, which can further exacerbate hypovolaemia and hypokalaemia.
Given that digoxin has a prolonged half-life with normal renal function, it is safe and probably reasonable to withhold digoxin for 1-2 days in severe gastroenteritis. While the stated therapeutic reference range is $0.6-2.6 \mathrm{nmol} / \mathrm{L}$, the current recommendation is to use lower doses and aim for a lower target concentration in the therapeutic range. ${ }^{29}$ The risk of toxicity from ongoing dosing is probably low in shortlived gastroenteritis.

\section{Oral contraception}

The loss of efficacy of the oral contraceptive pill (combined and progestogen-only) has been reported with diarrhoea and presumably relates to impaired absorption and sub-therapeutic hormone levels. Guidance for managing missed pills is applicable to intercurrent illness.

\section{Combined oral contraceptive pill}

If only one dose has been affected by the illness, the pill should be taken when symptoms stop and then the rest of the pack continued as usual. Two doses may need to be taken on the same day. No additional contraception is required. ${ }^{29}$

If the illness lasts for two or more pill-taking days, contraception will be affected. The last missed pill should be taken at the end of the illness and then the rest of the pack should be taken as usual. Barrier contraception is required for the next seven days. Active pills need to be taken for the next seven days after the illness to ensure contraception. This may require skipping the pill-free period and commencing the active pills of the next pack. ${ }^{29}$

\section{Progestogen-only pill}

If illness lasts longer than three hours, contraceptive efficacy will be affected and the next pill should be taken as soon as the illness concludes (this may mean taking two pills on the same day). Barrier contraception should be used for the next two days. ${ }^{30}$

\section{Other drugs}

There are many other drug classes where interruptions to therapy can have adverse therapeutic consequences (see Table). Continuing these medicines with an unchanged dosing regimen during a brief episode of acute illness is unlikely to predispose patients to adverse events. In a patient whose symptoms are persistent or severe enough to either cause a significant electrolyte derangement or acute kidney injury, early assessment of serum electrolytes and renal function may allow early detection and intervention.

People on chronic antimicrobial therapy such as antiretrovirals for HIV should continue these if possible. This reduces the risk of losing control of the infection and the potential emergence of antimicrobial resistance. 


\section{Conclusion}

Acute illness can result in significant changes to drug pharmacokinetics, which can either cause adverse drug events or potentiate the illness. In relatively healthy individuals, alterations in pharmacokinetics are usually transient and not clinically significant. However, some patients are at risk of a serious adverse event so it is important to identify them based on their medication regimen and comorbidities. Understanding the principles of pharmacokinetics and potential complications should help clinicians provide better information to patients and more comprehensive 'sick day' plans. This may improve long-term adherence and chronic disease management. $<$

Darren Roberts is the Chair of the Editorial Executive Committee of Australian Prescriber.

\section{REFERENCES}

1. Griffith K, Ashley C, Blakeman T, Fluck R, Lewington A, Selby N, et al. 'Sick day rules' in patients at risk of acute kidney injury: an interim position statement from the Think Kidneys Board. Version 6: July 2015. www.thinkkidneys.nhs.uk/ wp-content/uploads/2015/07/Think-Kidneys-Sick-Day-Rules-160715.pdf [cited 2017 Sep 1]

2. Hebbard G, Sun W, Bochner F, Horowitz M. Pharmacokinetic considerations in gastrointestinal motor disorders. Clin Pharmacokinet 1995:28:41-66. https://doi.org/10.2165/00003088-199528010-00005

3. Rangno RE, Langlois S, Lutterodt A. Metoprolol withdrawal phenomena: mechanism and prevention. Clin Pharmacol Ther 1982;31:8-15. https://doi.org/ 10.1038/clpt.1982.2

4. Gibb WR, Griffith DN. Levodopa withdrawal syndrome identical to neuroleptic malignant syndrome. Postgrad Med J 1986;62:59-60. https://doi.org/10.1136/ pgmj.62.723.59

5. National Clinical Guideline Centre (UK). Acute kidney injury: prevention, detection and management up to the point of renal replacement therapy. NICE Clinical Guidelines No. 169. London: Royal College of Physicians (UK); 2013.

6. Wheeler DC, Becker GJ. Summary of KDIGO guideline. What do we really know about management of blood pressure in patients with chronic kidney disease? Kidney Int 2013;83:377-83. https://doi.org/10.1038/ki.2012.425

7. Yacoub R, Patel N, Lohr JW, Rajagopalan S, Nader N, Arora P. Acute kidney injury and death associated with renin angiotensin system blockade in cardiothoracic surgery: a meta-analysis of observational studies. Am J Kidney Dis 2013;62:1077-86. https://doi.org/10.1053/j.ajkd.2013.04.018

8. Pretorius M, Murray KT, Yu C, Byrne JG, Billings FT, Petracek MR, et al. Angiotensin-converting enzyme inhibition or mineralocorticoid recepto blockade do not affect prevalence of atrial fibrillation in patients undergoing cardiac surgery. Crit Care Med 2012;40:2805-12. https://doi.org/10.1097/ CCM.0b013e31825b8be2

9. Cheungpasitporn W, Thongprayoon C, Srivali N, O'Corragain OA, Edmonds PJ, Ungprasert $\mathrm{P}$, et al. Preoperative renin-angiotensin system inhibitors use linked to reduced acute kidney injury: a systematic review and meta-analysis. Nephrol Dial Transplant 2015;30:978-88. https://doi.org/10.1093/ndt/gfv023

10. Dinsdale C, Wani M, Steward J, O’Mahony MS. Tolerability of spironolactone as adjunctive treatment for heart failure in patients over 75 years of age. Age Ageing 2005;34:395-8. https://doi.org/10.1093/ageing/afi104

11. Hieble JP, Ruffolo RR Jr. Possible structural and functional relationships between imidazoline receptors and alpha 2-adrenoceptors. Ann N Y Acad Sci 1995;763:8-21. https://doi.org/10.1111/j.1749-6632.1995.tb32387.x

12. Houston MC. Abrupt cessation of treatment in hypertension: consideration of clinical features, mechanisms, prevention and management of the discontinuation syndrome. Am Heart J 1981;102:415-30. https://doi.org/10.1016/ 0002-8703(81)90317-3

13. Rupp H, Maisch B, Brilla CG. Drug withdrawal and rebound hypertension: differential action of the central antihypertensive drugs moxonidine and clonidine. Cardiovasc Drugs Ther 1996;10 Suppl 1:251-62. https://doi.org/ 10.1007/BF00120495

14. Plänitz V. Crossover comparison of moxonidine and clonidine in mild to moderate hypertension. Eur J Clin Pharmacol 1984;27:147-52. https://doi.org/ 10.1007/BF00544037

15. Lilja M, Jounela AJ, Juustila HJ, Paalzow L. Abrupt and gradual change from clonidine to beta blockers in hypertension. Acta Med Scand 1982;211:375-80. https://doi.org/10.1111/j.0954-6820.1982.tb01965.x

16. Psaty BM, Koepsell TD, Wagner EH, LoGerfo JP, Inui TS. The relative risk of incident coronary heart disease associated with recently stopping the use of beta-blockers. JAMA 1990;263:1653-7. https://doi.org/10.1001/ jama.1990.03440120075040
17. Miller RR, Olson HG, Amsterdam EA, Mason DT. Propranolol-withdrawal rebound phenomenon. Exacerbation of coronary events after abrupt cessation of antianginal therapy. N Engl J Med 1975;293:416-8. https://doi.org/ 10.1056/NEJM197508282930902

18. Krukemyer JJ, Boudoulas H, Binkley PF, Lima JJ. Comparison of hypersensitivity to adrenergic stimulation after abrupt withdrawal of propranolol and nadolol: influence of half-life differences. Am Heart $J$ 1990;120:572-9. https://doi.org/10.1016/0002-8703(90)90013-N

19. Australian Diabetes Educators Association. Clinical guiding principles for sick day management of adults with type 1 and type 2 diabetes. Technical document. Canberra: Australian Diabetes Educator Association; 2016. https://www.adea.com.au/wp-content/uploads/2009/10/Clinical-GuidingPrinciples-for-Sick-Day-Management-of-Adults-with-type-1-and-type-2diabetes-Review-2016.docx.pdf [cited 2017 Sep 1]

20. The Royal Australian College of General Practitioners and Diabetes Australia. General practice management of type 2 diabetes - 2014-15. Melbourne: RACGP; 2014. https://static.diabetesaustralia.com.au/s/fileassets/diabetesaustralia/5ed214a6-4cff-490f-a283-bc8279fe3b2f.pdf [cited 2017 Sep 1]

21. Salpeter SR, Greyber E, Pasternak GA, Salpeter EE. Risk of fatal and nonfatal lactic acidosis with metformin use in type 2 diabetes mellitus. Cochrane Database Syst Rev 2010;14:CD002967. https://doi.org/10.1002/ 14651858.CD002967.pub4

22. Duong JK, Furlong TJ, Roberts DM, Graham GG, Greenfield JR, Williams KM, et al. The role of metformin in metformin-associated lactic acidosis (MALA): case series and formulation of a model of pathogenesis. Drug Saf 2013;36:733-46. https://doi.org/10.1007/s40264-013-0038-6

23. Lubowsky ND, Siegel R, Pittas AG. Management of glycemia in patients with diabetes mellitus and CKD. Am J Kidney Dis 2007;50:865-79. https://doi.org/ 10.1053/j.ajkd.2007.08.012

24. Harrower AD. Pharmacokinetics of oral antihyperglycaemic agents in patients with renal insufficiency. Clin Pharmacokinet 1996;31:111-9. https://doi.org/ 10.2165/00003088-199631020-00003

25. Degn KB, Brock B, Juhl CB, Djurhuus CB, Grubert J, Kim D, et al. Effect of intravenous infusion of exenatide (synthetic exendin-4) on glucose-dependent insulin secretion and counterregulation during hypoglycemia. Diabetes 2004:53:2397-403. https://doi.org/10.2337/diabetes.53.9.2397

26. Pendergrass M, Fenton C, Haffner SM, Chen W. Exenatide and sitagliptin are not associated with increased risk of acute renal failure: a retrospective claims analysis. Diabetes Obes Metab 2012;14:596-600. https://doi.org/10.1111/ j.1463-1326.2012.01567.x

27. Liu D, Ahmet A, Ward L, Krishnamoorthy P, Mandelcorn ED, Leigh R et al. A practical guide to the monitoring and management of the complications of systemic corticosteroid therapy. Allergy Asthma Clin Immunol 2013;9:30. https://doi.org/10.1186/1710-1492-9-30

28. Sick day rules for patients taking long-term steroid therapy (hydrocortisone, prednisolone \& cortisone acetate). Sick Day Rules 6.0.2. Norfolk and Norwich University Hospitals NHS Foundation Trust; 2016. http://www.nnuh.nhs.uk/ publication/sick-day-rules-6-0-2 [cited 2017 Sep 1]

29. Australian medicines handbook. Adelaide: Australian Medicines Handbook Pty Ltd; 2017. https://amhonline.amh.net.au [cited 2017 Sep 1]

30. NHS Choices. Contraception guide. What should I do if I miss a pill (progestogen-only pill)? Gov.UK; 2016. www.nhs.uk/Conditions/contraceptionguide/Pages/miss-progestogen-only-pill.aspx [cited 2017 Sep 1] 